\title{
Research Article \\ Effect of aerobic exercise training on anxiety in children with developmental coordination disorder
}

\author{
Sujatha B. ${ }^{1}$, Jagatheesan Alagesan ${ }^{2}$, R. Akahaya ${ }^{3}$, R. Rajameena ${ }^{3}$, A. Brite Saghaya Rayna ${ }^{4}$ \\ ${ }^{1} \mathrm{PhD}$ Scholar, ${ }^{2}$ Principal, ${ }^{3} \mathrm{BPT},{ }^{4} \mathrm{BPT}$ Final Year, Saveetha College of Physiotherapy, Saveetha Institute of Medical and \\ Technical Sciences, Chennai, Tamil Nadu, India
}

(Received: July $2020 \quad$ Revised: October $2020 \quad$ Accepted: November 2020)

Corresponding author: Jagatheesan Alagesan. Email: sujiphysio@gmail.com

\begin{abstract}
Introduction and Aim: Developmental Coordination Disorder (DCD) is a common neurodevelopmental disorder characterized by deficits in both fine and gross motor coordination which has a significant impact on a child's activities of daily living or school productivity. So, the study aims to find the effect of aerobic exercise training on anxiety in children with developmental coordination disorder. Only very few studies have so far examined the psychological benefits of aerobic exercise in children with DCD who are already at a higher risk for anxiety problems.

Materials and Methods: The school going children in Chennai district will be selected by convenient sampling method from the above-mentioned study setting based on the inclusion and exclusion criteria after a clear explanation about the study to the parents and teachers of the child with the inform consent an ascent will be obtained. Then the parents of the children are subjected to DCD-O7 questionnaire for the data collection.

Results: Statistical Analysis made with quantitative data revealed statistically significant difference between pre and post-test values. Results of this current study provide support for the beneficial effects of aerobic exercise on reducing anxiety in children population.

Conclusion: From the Result it has been concluded that the aerobic exercise showed the better result for reducing the increased level of anxiety in children with developmental coordination disorder. Hence this technique can be used for reducing anxiety of the DCD children and also to improve their quality of life, academic outcomes and physical activity. This study is the first study which has attempted to explore the relationship between aerobic exercise and anxiety in DCD Children.
\end{abstract}

Keywords: Anxiety; children; aerobic exercise

\section{INTRODUCTION}

$\mathrm{D}$ evelopmental Coordination Disorder (DCD) is a common neurodevelopmental disorder characterized by deficits in both fine and gross motor coordination which has a significant impact on a child's activities of daily living or school productivity. These deficits are present in the absence of severe intellectual or visual impairment, or another motor disability, such as cerebral palsy. It is thought to affect more than $5 \%$ of school-aged children $(1,2)$. Deficits in motor skills have been labelled in many ways over the centuries (e.g., neurological soft signs, clumsiness), but the current, dominant term is DCD (3).

DCD, sometimes referred to as dyspraxia affects between 2 and $6 \%$ of children (4) and is characterized by motor skills that are significantly below age-expected levels, persisting despite opportunities to acquire and develop these skills (5).

Both the International Statistical Classification of Diseases and Related Health Problems, $10^{\text {th }}$ Revision (ICD-10) (WHO, 2007b) and the DSM, Fifth Edition (DSM-V) (APA, 2013) outlines broad diagnostic criteria for the disorder. In the ICD-10, DCD, along with clumsy child syndrome and developmental dyspraxia, are subsumed under the heading Specific Developmental Disorder of Motor Function, and defined as "a disorder in which the main feature is a serious impairment in the development of motor coordination that is not solely explicable in terms of general intellectual retardation or of any specific congenital or acquired neurological disorder marked neurodevelopmental immaturities such as choreiform movements of unsupported limbs or mirror movements and other associated motor features, as well as signs of impaired fine and gross motor coordination" $(6,7)$.

DCD is described as a motor skill disorder, which is present when "the acquisition and execution of coordinated motor skills is substantially below that expected given the individuals' chronological age and opportunity for skill learning and use". The motor skill deficits of these children impact on everyday self-care (e.g., doing up snaps or zippers, brushing teeth, climbing stairs), academic performance (e.g., printing, using scissors, keyboarding, physical education class), and leisure 
activities (e.g., ball sports, bicycle riding). The underlying motor deficits vary across children and are still not fully understood however, they have significant impact on the child's ability to learn and perform everyday tasks that require coordination $(8,9)$. Aerobic exercises increase secretions of dopamine, serotonin and epinephrine which reduced anxiety respectively (10). The role of aerobic exercise has drawn significant attention in recent years for anxiety disorders in children.

Children with lower motor competence also demonstrate significantly poorer performance on important components of physical fitness, such as aerobic and anaerobic endurance and muscular strength, when compared against developing typically peers (10). So, the study aims to find the effect of aerobic exercise training on anxiety in children with developmental coordination disorder.

\section{MATERIALS AND METHODS}

Thirty subjects of school students were selected according to inclusion and exclusion criteria. Study type: single group quasi experimental study design. Study setting: sample is selected from a school at Chennai according to the inclusion and exclusion criteria. Sampling Technique: Random sampling technique. Sample Size: 30 subjects. Inclusion criteria: Both genders, Age group 9 to 14 years, and did not have any musculoskeletal disorders. Exclusion criteria: History of respiratory illness, cardiovascular diseases, acute trauma or fall, children who are already into treatment programs for anxiety and obesity.

Procedure: A total of 540 school going children in Chennai district were screened after prior permission was obtained from the school heads. A brief oral explanation was given to parents, teachers and children about the study and based on the inclusion and exclusion criteria children were recruited by convenient sampling method, informed consent and ascent was obtained. Parents or teachers of the children were asked to fill the DCD-O7 questionnaire. Children who scored 15- 57 in the DCDO7 Q and who also had anxiety disorder according to Spence children's anxiety scale (parents report) were taken as samples. On screening 540 children 13 children were found to be under DCD criteria with anxiety disorder among which there were 2 dropouts. $\operatorname{DSM}(V)$ has four criteria to rule out DCD. Those are: Criterion A - The acquisition and execution of coordinated motor skills is substantially below that expected given the individual's chronological age and opportunity for skill learning and use. Difficulties are maintained as clumsiness (eg., dropping or bumping into objects) as well as slowness and inaccuracy of performance of motor skills (eg., catching an object, using scissors or cutlery, handwriting, riding a bike or participating in sports).

Criterion B -The motor skills deficit in criterion A significantly and persistently interferes with activities of daily living appropriate to chronological age (eg., selfcare and self-maintenance) and effects academic / school productivity, prevocational and vocational activities, leisure and play.

Criterion $\mathrm{C}$-Onset of symptoms is in the early developmental period.

Criterion D -The motor skills deficits are not better explained by intellectual disability (intellectual developmental disorder) or visual impairment and are not attributable to a neurologic condition affecting movement (eg., cerebral palsy, muscular dystrophy, degenerative disorder).

\section{Treatment procedure}

The training protocol for DCD children with anxiety disorder consisted of Aerobic exercises for 8 weeks duration, single session per day (evening in the school playground) for 3 days/ week. At the end of the eighth week Post-test measurement was taken by using Spence children's anxiety scale (parents report). Each session consisted of 40 minutes. (5 minswarmup exercise, 30mins- aerobic training, 5minscool down exercise).

Mode: As the study population were children, the exercise session consisted of playful activities like brisk walking, skipping, running to music, treasure hunt, aerobic dance, hide and seek.

Intensity: $60-70 \%$ of heart rate (moderate Intensity) measured using heart rate monitor.

Duration: 8 weeks duration for single session per day for 3 days/ week.

Before the training session vital signs (heart rate, respiratory rate, blood pressure, temperature) of all children were recorded. Children with unstable vitals are excluded for that particular session alone.

Warmup phase: The warmup was carried out to prevent injuries, muscle cramps during the training session. It prepares the muscles for vigorous actions. The warmup phase consists of stretching exercise and light jogging for 5 minutes.

\section{Aerobic exercise phase}

Brisk walking: Brisk walking is a moderate-intensity exercise and has more benefits for fitness and reducing health risks than walking at an easy pace. Brisk walking is suggested to the respective subjects initially for about 5 minutes with the use of stopwatch and play cones, then gradually progressed to improve the aerobic exercise capacity and to reduce anxiety.

Skipping: Skipping helps to improve heart rate and blood pressure. Studies says that skipping helps to 
boost immune system, balance metabolism, and improve overall health in a short period of time. Moderate intensity of skipping was given for 5 minutes. Children were allowed to take rest if required.

Running to music: Running can significantly improve physical and mental health. With the help of play cones and stopwatch running to music was performed for the next 5 minutes.

Treasure hunt: Treasure hunt workout offers the opportunity to perform a variety of natural movement, it is performed by students for next 5 minutes.

Aerobic dance: It is a fun cardiovascular workout, keeping an active lifestyle will help increase endurance, reduce health risks. Aerobic dance increases the blood flow to your muscles and to lungs.

Hide and seek: The challenge of getting into the perfect hiding place will help improve their balance, agility, coordination and efficiency of movement.

Cooldown phase: Cooling down is an easy exercise, done after a more intense activity, to allow the body to gradually transition to a resting or near-resting state. Depending on the intensity of the exercise, cooling down can involve a slow jog or walk for 5 minutes. Cooling down allows the heart rate to return to its resting rate.

\section{Outcome measure}

\section{Developmental coordination disorder questionnaire (DCDQ'07):}

The Developmental Coordination Questionnaire (DCDQ) is a parent report measure developed to assist in the identification of Developmental Coordination Disorder (DCD) in children. Parents are asked to compare their child's motor performance to that of his/her peers using a 5-point Likert scale. It provides a standard method to measure a child's coordination in everyday, functional activities. The overall sensitivity is $84.6 \%$ and the specificity is $70.8 \%$.

\section{Spence children's anxiety scale (SCAS):}

The Spence Children's Anxiety Scale (SCAS) assesses the severity of anxiety symptoms, broadly and across six dimensions of anxiety, as proposed by the DSM-IV. School-aged versions are available for caregivers/parents and students. The scale can be used to evaluate the impact of therapy on anxiety symptoms in children and adolescents.

\section{RESULTS}

From statistical analysis made with the quantitative data it was found that there was significant difference between pre and post-test values. The data collection included 13 subjects in the study. The age group 9-14 years had 8 boys and 5 girls showed the statistically mean value in boys as 47.75 (SD 5.20) and in girls as 46.80 (SD 6.42). With significant $P$ value less than 0.0001 . This clearly shows that the boys were more prone (table 1 and 2). The comparison of spence children's anxiety scale in developmental coordination disorder children in the table 4 and graph 4 shows the statistically mean values in pretest as 62.18 (SD 7.39) and post- test as 49.27 (SD 4.20 ) and the t value was found to be 7.1466 with significant $\mathrm{P}$ value less than 0.0001 . This clearly shows that the posttest values were statistically significant (table 3 and 4).

Table 1: Comparison of DCD q-7 among children (Mean and SD values for DCD Q-7)

\begin{tabular}{|c|c|c|c|}
\hline Parameters & Mean & SD & $\begin{array}{c}\text { Number of } \\
\text { children } \\
\text { with DCD }\end{array}$ \\
\hline Boys & 47.75 & 5.20 & 8 \\
\hline Girls & 46.80 & 6.42 & 5 \\
\hline Combined & 47.38 & 5.45 & 13 \\
\hline
\end{tabular}

Table 2: Prevalence of DCD in children aged 9-14

\begin{tabular}{|c|c|c|c|c|c|c|c|}
\hline Age & $\mathbf{9}$ & $\mathbf{1 0}$ & $\mathbf{1 1}$ & $\mathbf{1 2}$ & $\mathbf{1 3}$ & $\mathbf{1 4}$ & Total \\
\hline Boys & 1 & 1 & 1 & 2 & 2 & 1 & 8 \\
\hline Girls & 1 & 0 & 1 & 1 & 2 & 0 & 5 \\
\hline Combined & 2 & 1 & 2 & 3 & 4 & 1 & 13 \\
\hline
\end{tabular}

Table 3: Number of samples included in the study

\begin{tabular}{|c|c|c|c|c|c|c|c|}
\hline Age & $\mathbf{9}$ & $\mathbf{1 0}$ & $\mathbf{1 1}$ & $\mathbf{1 2}$ & $\mathbf{1 3}$ & $\mathbf{1 4}$ & Total \\
\hline Boys & 1 & 1 & 1 & 2 & 2 & 0 & 7 \\
\hline Girls & 1 & 0 & 1 & 1 & 1 & 0 & 4 \\
\hline Combined & 2 & 1 & 2 & 3 & 3 & 0 & 11 \\
\hline
\end{tabular}

Table 4: Comparison of Spence children's anxiety scale in developmental coordination disorder of children: Pre and post-test values for Spence children's anxiety scale

\begin{tabular}{|c|c|c|c|c|c|}
\hline Parameter & Test & Mean & SD & $\begin{array}{c}\text { t } \\
\text { value }\end{array}$ & P value \\
\hline \multirow{2}{*}{ Anxiety } & $\begin{array}{c}\text { Pre- } \\
\text { test }\end{array}$ & 62.18 & 4.20 & \multirow{2}{*}{7.1466} & $<0.0001$ \\
\cline { 2 - 5 } & $\begin{array}{c}\text { Post- } \\
\text { test }\end{array}$ & 49.27 & 7.39 & & \\
\hline
\end{tabular}

\section{DISCUSSION}

The purpose of the study was to determine the effect of aerobic exercise training on anxiety in children with developmental coordination disorder. Several earlier studies have been directed to show the viability of aerobic exercise training strategies for anxiety. According to the DSM-V the prevalence of DCD in Thiruvallur district has been estimated to be between $3.8 \%$ in 9 to 14 years old children (10). DCD has been shown to be more prevalent in boys than girls, with reported sex ratios between 1.8 and five (10-12). 
Braaksma et al., reported that through affecting automatic neural system, aerobic exercises can reduce the level of stress and anxiety, which endanger the human health, and take a positive step toward reducing anxiety and depression (13). This may happen due to changes in cortisol level during doing aerobic exercises because various studies have reported the impact of aerobic exercise on reducing the level of cortisol and anxiety.

Faught et al, reported that when assessing the emotional wellbeing of children and adolescents (aged 10-14 years), those with poorer motor development reported lower perceived levels of competence (both scholastic and athletic), self worth, and social support than their peers, and they also endorsed greater levels of anxiety (14).

In line with predictions, parent reports demonstrated that children with DCD had elevated anxiety levels when compared with a matched, typically developing group. These findings support previous reports by Faught et al., and Dewey et al., $(3,14)$. Moreover, the findings of the current study extend these previous findings by focusing on individual differences within the group; which showed that many- but by no means all - individual children with a diagnosis of DCD had significantly elevated levels of anxiety. Skinner et al., reported that low perceptions of self worth in children and teenagers diagnosed with DCD varied with age and was not a whole group phenomenon, with perceptions changing as the child aged.

The Spence children's anxiety scale was used to find the anxiety in developmental coordination children. The pre intervention showed the mean of 62.18 and after the intervention was given the anxiety of the children reduced down to 49.27. The result clearly shows that there is beneficial effect in reducing anxiety level of DCD children and, hence increasing the aerobic capacity. The major limitation of the study is that we could not include many samples from the different geographical area and hence we would recommend in further study that we can include many subjects and also study the quality of life, their psycho social problems and physical fitness in children with developmental coordination disorder.

\section{CONCLUSION}

From the results it has been concluded that the moderate intensity aerobic exercise for 8 weeks in children with developmental coordination disorder has significantly reduced the anxiety level in these children. Hence aerobic exercises can be advised for reducing anxiety in DCD children which will in turn improve their quality of life, academic outcomes and physical activity.
Declared no conflict of interest

\section{REFERENCES}

1. Harrowell, I., Hollén, L., Lingam, R., Emond, A. The impact of developmental coordination disorder on educational achievement in secondary school. Research in developmental disabilities. 2018 Jan 1;72: 13-22.

2. American Psychiatric Association. Diagnostic and statistical manual of mental disorders (DSM-5®). American Psychiatric Pub, 2013.

3. Dewey, D., Kaplan, B. J. 0., Crawford, S. G., Wilson BN. Developmental coordination disorder: associated problems in attention, learning, and psychosocial adjustment. Human movement science. 2002 Dec 1; 21(5-6): 905-918.

4. Lingam, R., Hunt, L., Golding, J., Jongmans, M., Emond, A. Prevalence of developmental coordination disorder using the DSM-IV at 7 years of age: A UK population-based study. Pediatrics. 2009 Apr 1; 123(4): e693-e700.

5. Crane, L., Sumner, E., Hill, E. L. Emotional and behavioural problems in children with Developmental Coordination Disorder: Exploring parent and teacher reports. Research in Developmental Disabilities. 2017 Nov 1; 70: 67-74.

6. World Health Organization. International statistical classification of diseases and related health problems: 10th revision (ICD-10). http://www.WHO int/classifications/apps/icd/icd. 1992.

7. Blank, R., Barnett, A. L., Cairney, J., Green, D., Kirby, A., Polatajko, H., et al. International clinical practice recommendations on the definition, diagnosis, assessment, intervention, and psychosocial aspects of developmental coordination disorder. Developmental Medicine \& Child Neurology. 2019 Mar;61(3):242285.

8. Wilson, P. H., Ruddock, S., Smits-Engelsman, B., Polatajko., H. \& Blank, R. Understanding performance deficits in developmental coordination disorder: A meta-analysis of recent research. Developmental Medicine \& Child Neurology. 2012; 55: 217-228.

9. Missiuna, C., Campbell, W. N. Psychological aspects of developmental coordination disorder: can we establish casualty? Current Developmental Disorders Reports. 2014 Jun 1; 1(2): 125-131.

10. Long, B. C., Stavel, R. V. Effects of exercise training on anxiety: A meta-analysis. Journal of Applied Sport Psychology. 1995 Sep 1; 7(2): 167-89.

11. Sujatha, B., Jagatheesan Alagesan., D. V. La.1, and A. Brite Saghaya Rayna. Prevalence of Developmental Co-ordination Disorder in School Children. The Indian Journal of Pediatrics 2020; 87: 454-456.

12. Strauss, C. C., Frame, C. L., Forehand, R. Psychosocial impairment associated with anxiety in children. Journal of clinical child psychology. 1987 Sep 1; 16(3): 235239.

13. Braaksma, P., Stuive I., van der Hoek, F. D., Van Der Sluis, C. K., Schoemaker, M. M., Dekker, R. Improving Physical Fitness in 7-12-Year-Old Children with Developmental Coordination Disorder: Protocol of a Multicenter Single-Arm Mixed-Method Study. Frontiers in pediatrics. 2018 Dec 18; 6: 396.

14. Faught, B. E., Demetriades, S., Hay, J., Cairney, J. Does relative body fat influence the Movement ABC-2 assessment in children with and without developmental coordination disorder? Research in developmental disabilities. 2013 Dec 1; 34(12): 4433-4438.

\section{CONFLICT OF INTEREST}

